\title{
Overnight/first-morning urine free metanephrines and methoxytyramine for diagnosis of pheochromocytoma and paraganglioma: is this an option?
}

Mirko Peitzsch', Denise Kaden', Christina Pamporaki², Katharina Langton², Georgiana Constantinescu², Catleen Conrad' ${ }^{1}$, Stephanie Fliedner ${ }^{3}$, Richard 0 Sinnott ${ }^{4}$, Aleksander Prejbisz ${ }^{5}$, Roland Därr ${ }^{6}$, Jacques W M Lenders ${ }^{2,7}$, Michael Bursztyn ${ }^{\mathbf{8}}$ and Graeme Eisenhofer ${ }^{1,2}$

${ }^{1}$ Institute of Clinical Chemistry and Laboratory Medicine, University Hospital Carl Gustav Carus, Technische Universität Dresden, Dresden, Germany, ${ }^{2}$ Department of Medicine III, University Hospital Carl Gustav Carus, Technische Universität Dresden, Dresden, Germany, ${ }^{3}$ First Department of Medicine, University Medical Center Schleswig-Holstein, Lübeck, Germany, ${ }^{4}$ School of Computing and Information Systems, University of Melbourne, Melbourne, Australia, ${ }^{5}$ Department of Hypertension, Institute of Cardiology, Warsaw, Poland, 'Department of Medicine IV, Faculty of Medicine, University of Freiburg, Freiburg, Germany, ${ }^{7}$ Department of Internal Medicine, Radboud University Medical Centre, Nijmegen, The Netherlands, and ${ }^{8}$ Department of Medicine, Hypertension Unit, Hadassah-Hebrew University Medical Center, Jerusalem, Israel

Correspondence should be addressed to $\mathrm{G}$ Eisenhofer Email

graeme.eisenhofer@ uniklinikum-dresden.de

\begin{abstract}
Objective: Sympathoadrenal activity is decreased during overnight rest. This study assessed whether urinary-free normetanephrine, metanephrine and methoxytyramine in overnight/first-morning urine collections might offer an alternative to measurements in 24-h collections or plasma for diagnosis of pheochromocytoma and paraganglioma (PPGL).

Design and methods: Prospective multicenter cross-sectional diagnostic study involving 706 patients tested for PPGL, in whom tumors were confirmed in 79 and excluded in 627 after follow-up. Another 335 age- and sex-matched volunteers were included for reference purposes. Catecholamines and their free O-methylated metabolites were measured in 24-h collections divided according to waking and sleeping hours and normalized to creatinine. Plasma metabolites from blood sampled after supine rest were measured for comparison.

Results: Urinary outputs of norepinephrine, normetanephrine, epinephrine and metanephrine in the reference population were respectively 50 (48-52)\%, 35 (32-37)\%, 76 (74-78)\% and 15 (12-17)\% lower following overnight than daytime collections. Patients in whom PPGLs were excluded showed 28 (26-30)\% and 6 (3-9)\% day-to-night falls in normetanephrine and metanephrine, while patients with PPGLs showed no significant day-to-night falls in metabolites. Urinary methoxytyramine was consistently unchanged from day to night. According to receiver-operating characteristic curves, diagnostic accuracy of metabolite measurements in overnight/first-morning urine samples did not differ from measurements in 24-h urine collections, but was lower for both than for plasma. Using optimized reference intervals, diagnostic specificity was higher for overnight than daytime collections at similar sensitivities. Conclusions: Measurements of urinary-free catecholamine metabolites in first-morning/overnight urine collections offer an alternative for diagnosis of PPGL to 24-h collections but remain less accurate than plasma measurements.
\end{abstract}




\section{Introduction}

The Endocrine Society (USA) guidelines recommend urinary or plasma metanephrines (i.e. normetanephrine and metanephrine) for diagnosis of pheochromocytoma and paraganglioma (PPGL), recognizing their relatively specific sources from within chromaffin cells and tumor derivatives and thereby their superior accuracy for diagnosis over the parent catecholamines and other metabolites (1). Urinary metanephrines are, however, commonly measured after an acid-hydrolysis deconjugation step and largely represent sulfateconjugated metabolites produced at different sites in the body from the free metanephrines (2). Superior diagnostic accuracy of the free metanephrines compared to the deconjugated metabolites has been confirmed for plasma measurements (3). However, at the time of publication of the Endocrine Society Guidelines (1), it was not clear whether measurements of the free metabolites in plasma provided superior diagnostic accuracy over measurements of the deconjugated metabolites in urine. Inferior diagnostic accuracy of urinary deconjugated metanephrines compared to both plasma and urinary-free metanephrines has since been established in a prospective study involving over 2000 patients (4). That study also included measurements of methoxytyramine, the O-methylated metabolite of dopamine, which although useful in plasma, exhibits limited diagnostic utility in urine, whether free or deconjugated.

As the activation of sympathoadrenal systems increases secretion of catecholamines and production of their metabolites, it is important that such activation is avoided during diagnostic testing for PPGLs; in particular, blood samples should be collected after supine rest $(5,6,7,8)$. Without such precautions, there is little if any diagnostic advantage of plasma over urine tests (6); the only remaining advantage is the simpler and more rapid procedure of blood sampling compared to 24-h urine collections. The latter are inconvenient for patients and also susceptible to inaccuracy due to incomplete or incorrect collections. Twenty-four-hour urine collections also do not easily control for increases in sympathoadrenal activity associated with daily life stressors. Influences of diet, which can increase plasma and urinary methoxytyramine and deconjugated normetanephrine (9), can also contribute to false-positive results. For the plasma test, dietary influences can be minimized by an overnight fast before blood sampling. For urine collections, daily influences that increase sympathoadrenal activity may potentially be avoided by collecting urine after a period of overnight rest. Indeed, it is well-established that plasma concentrations and urinary outputs of norepinephrine and epinephrine are lower when sampled after overnight rest or during sleep than in daytime collections $(10,11,12,13)$.

Based on the aforementioned considerations, it was hypothesized that measurements of free metanephrines and methoxytyramine in overnight collections of urine would offer similar performance to the plasma test for diagnosis of PPGLs and improved accuracy compared to measurements in 24-h collections. This hypothesis was addressed in the present study, a sub-study of the prospective monoamine-producing tumor (PMT) study (4), by the collection of urine samples over 24-h that were split into two collection periods, the first during daytime waking hours and the second during overnight rest. Urinary catecholamines were also measured to verify impacts of overnight rest on sympathoadrenal function and thereby provide reference comparators for downstream impacts on the metabolites.

\section{Subjects and methods}

\section{Subjects}

A total of 706 patients (348 males) at a median age of 54 years (range 13-85) and with suspicion or at risk for PPGLs were enrolled into this cross-sectional diagnostic study under a multicenter prospective clinical protocol described in the manuscript and supplement of the main study published elsewhere (4). These 706 patients represented a subset of over 2000 patients enrolled into the PMT study for whom separate day and overnight urine collections were available and consecutively analyzed for daytime and overnight urinary outputs of metabolites. In brief, there were four main criteria for establishing suspicion or risk for PPGLs: (1.) signs and/or symptoms of presumed catecholamine excess; (2.) hereditary risk of PPGL; (3.) findings of an abdominal or adrenal incidentaloma or (4.) previous history of PPGL. The study contained multiple phases, from screening to follow-up, according to an Ethics committee-approved clinical protocol and standard-operating procedures available online (https:// pmt-study.pressor.org).

In the 706 patients tested for PPGLs, tumors were confirmed in 79 and excluded in 627 patients (Table 1). Confirmation usually involved histopathological examination of resected tumors, but occasionally depended on functional imaging evidence (e.g. for metastatic 
disease), while exclusion involved follow-up according to criteria and procedures described previously (4). Among the 79 patients with PPGLs, 61 had pheochromocytomas and 17 had paragangliomas, including head and neck paragangliomas in 6 patients. A reference population of 335 normotensive and hypertensive volunteers with a median age of 51 years (range 18--82), recruited at Dresden and matched according to age and sex with the patient population, was included for comparison and for establishing reference intervals. The clinical protocols under which patients or volunteers were enrolled were approved by the Ethics Committees of the University Hospital Dresden, the Radboud University Medical Center, the University Medical Center Schleswig-Holstein and the Institute of Cardiology at Warsaw, these comprising the four centers that contributed subjects to the presently described PMT sub-study. All subjects provided written, informed consent before study inclusion.

\section{Urine and blood collections}

Urine collections were split into daytime and overnight collections. This was achieved for daytime collections by excluding the first-morning urine collection and continuing collections until inclusion of the last void before overnight recumbent rest or sleep. Overnight collections included all subsequent collections up until and including the first-morning urine. Daytime and overnight urinary outputs were summed to provide 24-h urine outputs. Urine specimens were returned to study centers on the final day of collection, at which time volumes were determined and samples aliquoted for storage at $-80^{\circ} \mathrm{C}$. Blood samples were taken in the morning hours after an overnight fast, by maintaining the supine position for 30 min up until the time when blood was drawn. Blood samples were kept cool at $4^{\circ} \mathrm{C}$ before centrifugation to separate the plasma. All specimens collected at different study centers were shipped to the central analytical laboratory on dry ice, where the biochemical analyses were performed.

\section{Biochemical analyses}

Analyses of plasma-free metanephrines and 3-methoxytyramine and urinary-free metanephrines, methoxytyramine and catecholamines (norepinephrine, epinephrine and dopamine) were performed at a single laboratory using liquid chromatographytandem mass spectrometry (LC-MS/MS) according to previously described and validated methods $(14,15,16)$. Measurements in 24-h urine collections are expressed in $\mathrm{nmol}$ per day (nmol/day), whereas those in split day and overnight urine collections were normalized for differences in dilution according to outputs of creatinine and are expressed as nmol per mmol creatinine $(\mathrm{nmol} / \mathrm{mmol})$. Urinary creatinine concentrations were determined on a Cobas 8000 analyzer (Roche Diagnostics). The reference intervals used to distinguish elevated from normal test results for plasma metabolites and 24-h urinary outputs of free metabolites have been described elsewhere $(4,17)$, whereas those for day and overnight collections of urine normalized to creatinine are established here.

\section{Statistical analysis}

Statistical analyses utilized the JMP software package (SAS Institute Inc, Cary, NC). Differences between groups in plasma concentrations and urinary outputs of analytes were established using the Kruskal-Wallis test, with the Steel-Dwass test employed for post-hoc analyses. Differences between daytime and overnight collections were assessed using the paired Wilcoxon signrank sum test, while the significance of relationships between variables was assessed using Spearman's rank correlation coefficient. Differences between groups in changes from daytime to overnight collections were assessed by parametric analyses after first normalizing ratios of overnight to daytime collections by logarithmic transformation. Data from those analyses were then expressed as geometric means and 95\% confidence intervals (95\% CIs).

Differences in the performance of diagnostic tests were primarily assessed from receiver-operating characteristic (ROC) curves constructed using logistic regression. Comparisons of areas-under-curves (AUCs) to assess the significance of differences, including estimation of $95 \%$ CIs of AUCs and 95\% CIs of differences between AUCs, utilized the JMP model comparison function. Differences in test performance were also assessed from comparisons of diagnostic sensitivity and specificity according to reference intervals, established here for day and overnight collections from distributions of data for the reference population. Consistent with previous considerations (17), upper cut-offs for normetanephrine utilized the 97.5 percentiles. In contrast, recognizing the secondary importance of the other two metabolites, reference intervals for metanephrine and methoxytyramine utilized 
the 99.5 percentiles. With those cut-offs, diagnostic sensitivity was estimated from the percentage of truepositive results among both true-positive and falsenegative results for patients with PPGLs. Diagnostic specificity was estimated from the percentage of truenegative results among both true-negative and falsepositive results. Differences in sensitivity or specificity between tests were assessed using McNemar's test and from 95\% CIs of differences (Supplementary Table 1, see section on supplementary materials given at the end of this article).

\section{Results}

\section{Plasma and 24-h urinary outputs of catecholamine metabolites}

Plasma concentrations and 24-h urinary outputs of free normetanephrine and methoxytyramine did not differ between patients tested for PPGLs in whom tumors were excluded and for subjects of the age-matched and sex-matched reference population (Table 1 ). However, plasma concentrations and 24-h urinary excretion of free metanephrine were $13-14 \%$ lower $(P<0.02)$ in the former than the latter group, this reflecting inclusion of some patients with past histories of adrenalectomies due to pheochromocytoma. Patients with confirmed PPGL showed 7.7-, 4.4- and 2.7-fold higher $(P<0.0001)$ plasma concentrations of normetanephrine, metanephrine and methoxytyramine and 9.3-, 5.1- and 1.6-fold higher $(P<0.001)$ 24-h urinary outputs of the same respective metabolites than patients in whom disease was excluded.

\section{Daytime and overnight urinary catecholamines and metabolites}

Daytime and overnight urinary outputs of norepinephrine were respectively 3.6- and 3.7-fold higher $(P<0.0001)$ in patients with PPGLs than without PPGLs, compared to larger 9.5- and 11.9-fold higher $(P<0.0001)$ outputs for normetanephrine (Table 1). Daytime and overnight urinary outputs for epinephrine were respectively 3.2- and 7.3-fold higher $(P<0.0001)$ in patients with PPGLs than without PPGLs, compared to 4.1- and 4.7-fold $(P<0.0001)$ higher outputs for metanephrine. In contrast, daytime and overnight urinary outputs for dopamine were only 1.2fold higher $(P<0.02)$ in patients with PPGLs than without
PPGLs, while urinary outputs of methoxytyramine were 1.5-fold higher $(P<0.0001)$.

Urinary outputs of norepinephrine, epinephrine, normetanephrine and metanephrine all showed day-tonight decreases $(P<0.0001)$ in the reference population and patients in whom PPGLs were excluded (Table 1). In contrast, urinary outputs of normetanephrine and metanephrine showed no significant day-to-night decreases in patients with PPGLs, though there were decreases $(P<0.0001)$ in urinary norepinephrine and epinephrine. Except for small day-to-night decreases $(P<0.0001)$ in urinary dopamine in reference subjects, there were no significant day-to-night differences for dopamine in the other two groups and no day-to-night differences for methoxytyramine in any group.

\section{Day-to-night differences between groups}

The largest day-to-night falls in urinary excretion of catecholamines and their metabolites were observed for epinephrine (76\%) and norepinephrine (50\%) in the reference population (Fig. 1). These decreases were larger $(P<0.0001)$ than respective day-to-night decreases for both patients in whom PPGLs were excluded $(66 \%$ and $44 \%$ ) and confirmed (43\% and 30\%). Urinary excretion of free normetanephrine and metanephrine showed similar patterns of day-to-night differences among the three groups to the parent catecholamines, but for all groups day-to-night differences were smaller $(P<0.0001)$ for the metabolites than for the parent catecholamines. Urinary excretion of metanephrine, in particular, showed day-tonight respective decreases in the reference group and in patients without PPGLs of only $15 \%$ and $6 \%$ compared to $76 \%$ and $66 \%$ for epinephrine.

\section{Day-to-night decreases vs increases in catecholamines and their metabolites}

Day-to-night changes in normetanephrine and metanephrine were positively related $(P<0.0001)$ to changes in their respective parent catecholamines for each of the three groups of subjects (Fig. 2). Proportions of patients showing day-to-night decreases in catecholamines and their metabolites predominated over those showing increases; this was particularly evident for subjects of the reference group in whom day-to-night respective increases in norepinephrine, epinephrine and normetanephrine were observed in only 3.6\%, 2.1\% and 
Table 1 Patient demographics and catecholamine-related biochemical variables in the reference population and patients tested for PPGLs in whom tumors were excluded vs confirmed.

\begin{tabular}{|c|c|c|c|}
\hline & Reference & PPGL excluded & PPGL confirmed \\
\hline \multicolumn{4}{|l|}{ Demographics } \\
\hline$n$ & 335 & 627 & 79 \\
\hline $\operatorname{Sex}(F / M)$ & $173 / 162$ & $312 / 315$ & $46 / 33$ \\
\hline Age mean (range) & $50(18-82)$ & $52(13-85)$ & $49(14-75)$ \\
\hline \multicolumn{4}{|c|}{ Plasma free metabolites (nmol/L) } \\
\hline Normetanephrine & $0.35(0.28-0.46)$ & $0.39(0.27-0.53)$ & $3.02(1.08-7.70)^{* \star \dagger}$ \\
\hline Metanephrine & $0.16(0.12-0.20)$ & $0.14(0.10-0.19)^{*}$ & $0.62(0.17-2.40)^{* * \dagger}$ \\
\hline Methoxytyramine & $0.03(0.02-0.04)$ & $0.03(0.02-0.05)$ & $0.08(0.05-0.18)^{* *^{\dagger}}$ \\
\hline \multicolumn{4}{|c|}{ Urinary free metabolites (nmol/day) } \\
\hline Normetanephrine & $117(90-157)$ & $121(88-168)$ & $1124(431-2682)^{\star \star \dagger}$ \\
\hline Metanephrine & $86(59-119)$ & $74(49-108)^{*}$ & $374(93-1638)^{* \star \dagger}$ \\
\hline Methoxytyramine & $190(146-250)$ & 198 (149-272) & $309(236-455)^{\star * \dagger}$ \\
\hline \multicolumn{4}{|c|}{ Daytime and overnight urinary excretion of catecholamines ( $\mathrm{nmol} / \mathrm{mmol}$ creatinine) } \\
\hline Day norepinephrine & $15.4(10.9-20.7)$ & $15.0(10.1-21.5)$ & $55.0(23.5-112.9)^{* * \dagger}$ \\
\hline Night norepinephrine & $7.7(5.2-11.3)$ & $8.6(5.7-13.6)^{\star}$ & $30.9(14.5-106.4)^{* * \dagger}$ \\
\hline$P$-value (day-to-night) & $<0.0001$ & $<0.0001$ & 0.0003 \\
\hline Day epinephrine & $3.1(2.1-4.4)$ & $2.5(1.4-3.9)^{\star *}$ & $8.1(2.7-42.7)^{\star \star \star t}$ \\
\hline Night epinephrine & $0.7(0.5-1.1)$ & $0.8(0.5-1.3)$ & $5.8(0.9-35.1)^{* \star \dagger}$ \\
\hline$P$-value (day-to-night) & $<0.0001$ & $<0.0001$ & $<0.0001$ \\
\hline Day dopamine & $125(101-159)$ & $117(88-153)^{*}$ & $145(108-190)^{* \dagger}$ \\
\hline Night dopamine & $120(95-148)$ & $120(89-159)$ & $140(101-187)^{\star \S}$ \\
\hline$P$-value (day-to-night) & $<0.0001$ & 0.8713 & 0.2533 \\
\hline \multicolumn{4}{|c|}{ Daytime and overnight urinary excretion of free metabolites ( $\mathrm{nmol} / \mathrm{mmol}$ creatinine) } \\
\hline Day normetanephrine & $12.0(8.7-15.6)$ & $12.5(8.9-17.1)$ & $118.7(41.9-240.4)^{\star * \dagger}$ \\
\hline Night normetanephrine & $7.7(5.7-10.6)$ & $8.9(6.3-12.8)^{\star \star}$ & $105.5(35.8-284.3)^{\star * \dagger}$ \\
\hline$P$-value (day-to-night) & 0.0001 & $<0.0001$ & 0.1134 \\
\hline Day metanephrine & $8.2(5.5-10.5)$ & $6.9(4.8-9.6)^{*}$ & $28.5(8.1-153.5)^{\star \star \dagger}$ \\
\hline Night metanephrine & $6.8(3.5-9.2)$ & $6.3(4.5-9.2)$ & $29.6(7.0-137.3)^{* *^{\dagger}}$ \\
\hline$P$-value (day-to-night) & $<0.0001$ & $<0.0001$ & 0.4006 \\
\hline Day methoxytyramine & $17.0(13.3-21.1)$ & $18.3(14.3-23.1)^{*}$ & $26.4(18.4-47.0)^{* \star \dagger}$ \\
\hline Night methoxytyramine & $16.9(13.3-20.5)$ & $18.3(14.3-23.8)^{* *}$ & $27.7(17.9-45.7)^{\star \star \dagger \dagger}$ \\
\hline$P$-value (day-to-night) & 0.8396 & 0.8271 & 0.8960 \\
\hline
\end{tabular}

All results for catecholamines and catecholamine metabolites are shown as medians and interquartiles.

${ }^{*} P P 0.0001, * P<0.02$, different from reference group; ${ }^{\dagger} P<0.0001,{ }^{5} P<0.005$, different from PPGL excluded group. The significance of differences in urinary outputs from daytime to overnight collections (day to night) were assessed by Wilcoxon's paired signed-rank sum test and are shown as $P$-values.

$6.6 \%$ of subjects. In contrast, among patients in whom PPGLs were excluded, the proportions of patients showing day-to-night increases were doubled for normetanephrine and 3.7- and 3.0-fold higher for norepinephrine and epinephrine.

\section{Reference intervals for overnight and daytime collections of metabolites}

Overnight and daytime urinary outputs of normetanephrine normalized to creatinine were respectively $26 \%$ and $38 \%$ higher $(P<0.0001)$ in women than men of the reference population, while urinary excretion of methoxytyramine was $26 \%$ higher $(P<0.0001)$ for both collections in women than in men. Excretion of metanephrine was $9 \%$ and $8 \%$ higher in women than men, but this was only significant $(P=0.0290)$ for daytime collections. Upper cut-offs of reference intervals for excretion of normetanephrine, defined by the $97.5 \%$ percentiles, were thus $12-20 \%$ higher in women than men and $65-76 \%$ higher for both sexes for daytime than overnight collections (Table 2). In contrast, the 97.5 and 99.5 percentiles of distributions for metanephrine and methoxytyramine showed either little or highly variable differences according to sex and daytime vs overnight collections.

\section{Diagnostic test performance}

Comparisons of ROC curves established higher $(P<0.05)$ AUCs for plasma measurements of free normetanephrine, metanephrine and methoxytyramine than urinary measurements of the metabolites in both $24-\mathrm{h}$ and overnight urine collections (Fig. 3). AUCs did not differ 

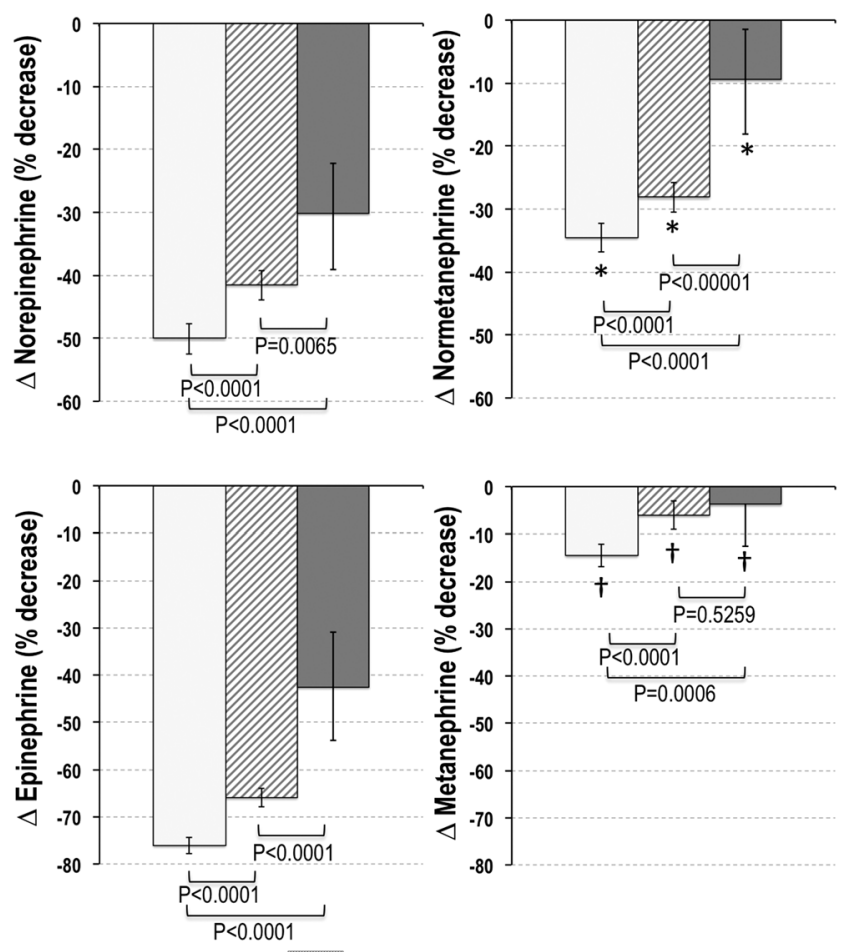

Reference

PPGL excluded

PPGL confirmed

\section{Figure 1}

Percent day-to-night decreases in urinary outputs of free norepinephrine, normetanephrine, epinephrine and metanephrine in subjects of the reference population and patients in whom PPGLs were excluded and confirmed. All data are shown as geometric means with confidence intervals. $\star P<0.0001$ lower day-to-night fall in urinary normetanephrine compared to norepinephrine; ${ }^{\dagger} P<0.0001$ lower day-to-night fall in urinary metanephrine compared to epinephrine.

for tests involving 24-h and overnight collections of metabolites. The AUC for urinary metabolites was higher $(P=0.0087)$ than for urinary catecholamines and was not increased by combining urinary catecholamines with metabolites.

Using previously validated reference intervals for free normetanephrine, metanephrine and methoxytyramine in plasma and 24-h collections of urine $(4,17)$, the plasma test demonstrated higher $(P=0.0047)$ diagnostic sensitivity than the urine test at identical specificities (Table 3). With the use of daytime and overnight sexspecific upper cut-offs, derived from 97.5 percentiles for urine-free normetanephrine and 99.5 percentiles for metanephrine and methoxytyramine, the diagnostic sensitivity for metabolites was also lower $(P=0.0143)$ for daytime collections of urine than for plasma, but not for overnight collections. However, measurements
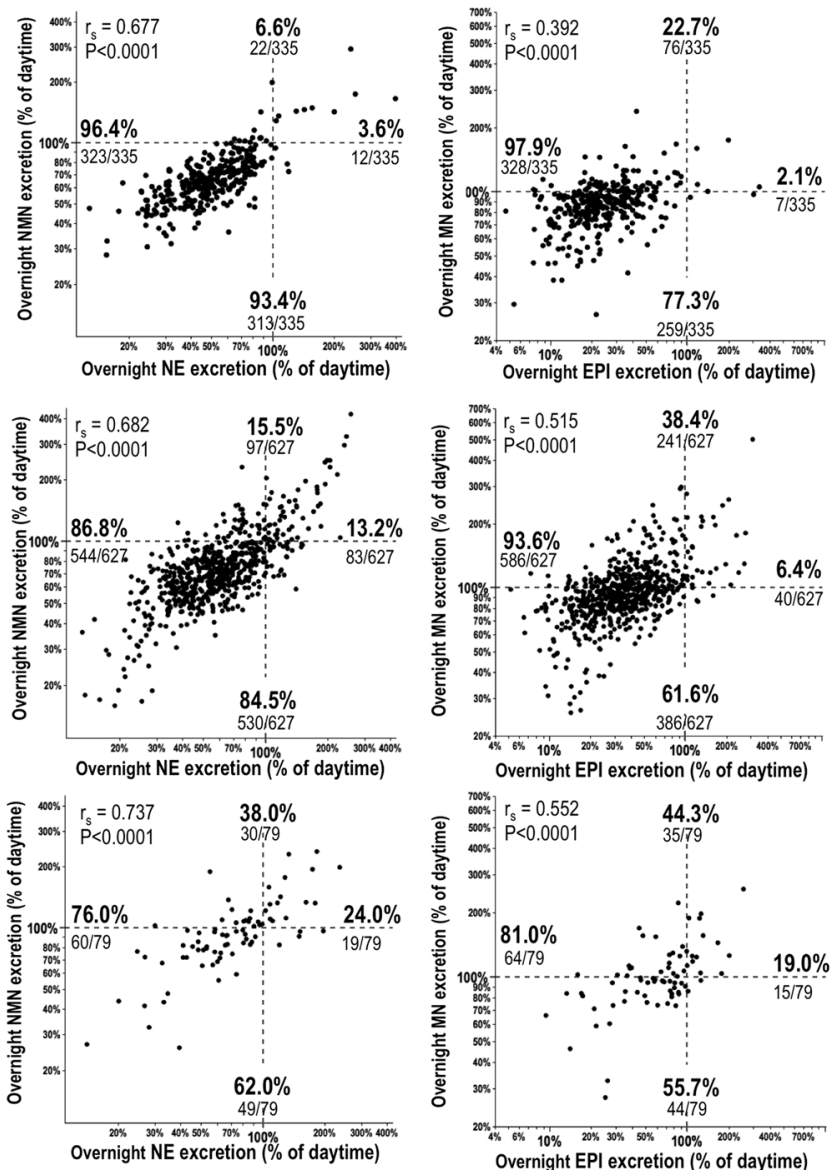

\section{Figure 2}

Relationships of day-to-night differences in urinary excretion normetanephrine (NMN) with norepinephrine (NE) in left panels and of metanephrine (MN) with epinephrine (EPI) in right panels for the reference group (upper panels) and patients in whom PPGLs were excluded (middle panels) or confirmed (lower panels). Dashed horizontal and vertical lines demark where subjects show day-to-night increases vs decreases in respective metanephrines and catecholamines. Proportions of patients with day-to-night increases vs decreases in urinary excretion of NMN or MN are shown in respective upper and lower portions of each panel, whereas proportions of patients with day-to-night increases vs decreases in urinary excretion of NE or EPI are shown in respective right and left portions of each panel.

of metabolites in overnight collections of urine showed lower $(P<0.0001)$ specificity than measurements in both plasma and 24-h collections, but with higher $(P=0.0253)$ sensitivity than for urinary $24-\mathrm{h}$ collections. Similarly, measurements of metabolites in daytime collections of urine also showed lower $(P=0.0219)$ specificity than both plasma and 24-h collections. 
Table 2 Reference intervals for daytime, overnight and combined day and night urine free metabolites. Reference intervals are shown separately for male and female adults in units of nmol of metabolite per mmol creatinine and were determined according to the 97.5 and 99.5 percentiles of distributions for the 335 subjects of the reference population.

\begin{tabular}{|c|c|c|}
\hline & Females & Males \\
\hline \multicolumn{3}{|c|}{ Free normetanephrine ( 2.5 and 97.5 percentiles) } \\
\hline Day & $5.3-31.4^{a}$ & $4.8-26.2^{a}$ \\
\hline Night & $4.1-17.8^{a}$ & $3.3-15.9^{a}$ \\
\hline Combined & $5.0-24.7^{b}$ & $4.5-21.0^{\mathrm{b}}$ \\
\hline \multicolumn{3}{|c|}{ Free metanephrine (2.5 and 97.5 percentiles) } \\
\hline Day & 2.3-19.4 & $2.7-16.1$ \\
\hline Night & $2.2-14.9$ & $2.1-14.2$ \\
\hline Combined & $2.5-18.9$ & $2.9-16.1$ \\
\hline \multicolumn{3}{|c|}{ Free methoxytyramine ( 2.5 and 97.5 percentiles) } \\
\hline Day & $7.6-40.5$ & $8.2-33.3$ \\
\hline Night & $8.1-33.2$ & $7.9-33.3$ \\
\hline Combined & $7.5-32.2$ & $7.8-30.3$ \\
\hline \multicolumn{3}{|c|}{ Free normetanephrine (0.5 and 99.5 percentiles) } \\
\hline Day & $4.2-54.3$ & $3.9-33.2$ \\
\hline Night & $3.0-28.3$ & $0.5-27.5$ \\
\hline Combined & $3.8-45.3$ & $3.6-30.8$ \\
\hline \multicolumn{3}{|c|}{ Free metanephrine ( 0.5 and 99.5 percentiles) } \\
\hline Day & $1.6-21.7^{a}$ & $1.8-24.5^{a}$ \\
\hline Night & $1.9-24.1^{\mathrm{a}}$ & $2.0-17.8^{a}$ \\
\hline Combined & $1.9-23.5^{b}$ & $2.0-22.9^{b}$ \\
\hline \multicolumn{3}{|c|}{ Free methoxytyramine (0.5 and 99.5 percentiles) } \\
\hline Day & $3.1-58.1$ & $6.2-40.9$ \\
\hline Night & $4.9-43.6$ & $6.9-43.2$ \\
\hline Combined & $3.5-42.4^{a, b}$ & $6.6-35.9^{a, b}$ \\
\hline
\end{tabular}

Indicates day and night and sex-specific upper cut-offs used to establish diagnostic performance in Table 3; b Indicates sex-specific cut-offs from combined day-and-night collections used to establish diagnostic performance in Table 3.

Optimized diagnostic performance for measurements of metabolites in overnight urine collections was achieved using sex-specific upper cut-offs derived from both combined daytime and overnight urine collections (Table 3). Using these upper cut-offs, measurements of metabolites in overnight urine collections yielded a diagnostic sensitivity of $93.7(88.2-99.2) \%$ and a specificity of 94.3 (92.4-96.1)\%, only marginally lower than the sensitivity of 97.5 (93.9-100.0)\% and specificity of 94.7 (93.0-96.5)\% for plasma measurements. Importantly, the sensitivity for overnight collections was higher $(P=0.0253)$ than for 24 -h urine collections at a near equivalent specificity. Moreover at these optimized cut-offs, although sensitivity did not differ between overnight and daytime urine collections, specificity was higher $(P<0.0001)$ for overnight than for daytime collections.

\section{Discussion}

Although measurements of free metanephrines and methoxytyramine in overnight or first-morning urine collections were not as accurate as plasma measurements for diagnosis of PPGL, this study does indicate that overnight urine collections may offer a viable alternative to 24-h collections. Such an alternative minimizes problems for patients associated with 24-h collections and may be useful for centers where the plasma test is unavailable or where it is cost-prohibitive or otherwise not feasible to collect blood after the recommended 20-min minimum supine rest period. The latter is particularly relevant for certain healthcare systems, such as in Britain, where the cost of blood sampling in the supine position has been estimated to be nearly 15 -fold higher than for seated sampling (18).

The aforementioned considerations are important when it is appreciated that the high diagnostic accuracy of the plasma test requires blood sampling after supine rest $(5,6,7,8)$. Without supine rest and other precautions, diagnostic accuracy of the plasma test is no better than the urinary test (6). This is because upright posture is associated with increased sympathoneuronal release of norepinephrine to maintain blood pressure $(19,20$, $21)$; thus sampling of blood in the seated, compared to 
A

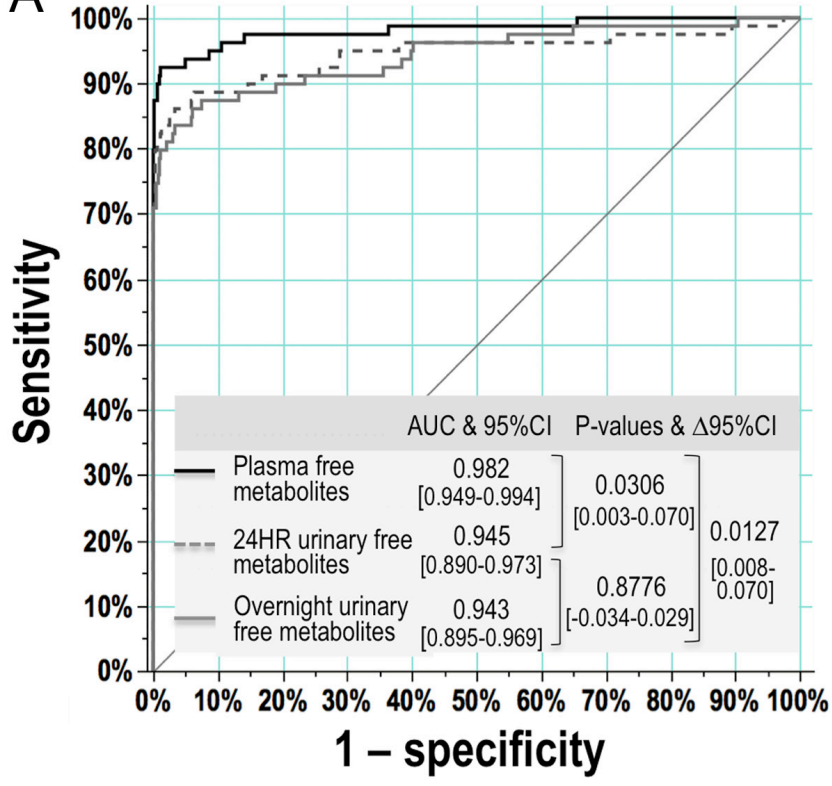

B

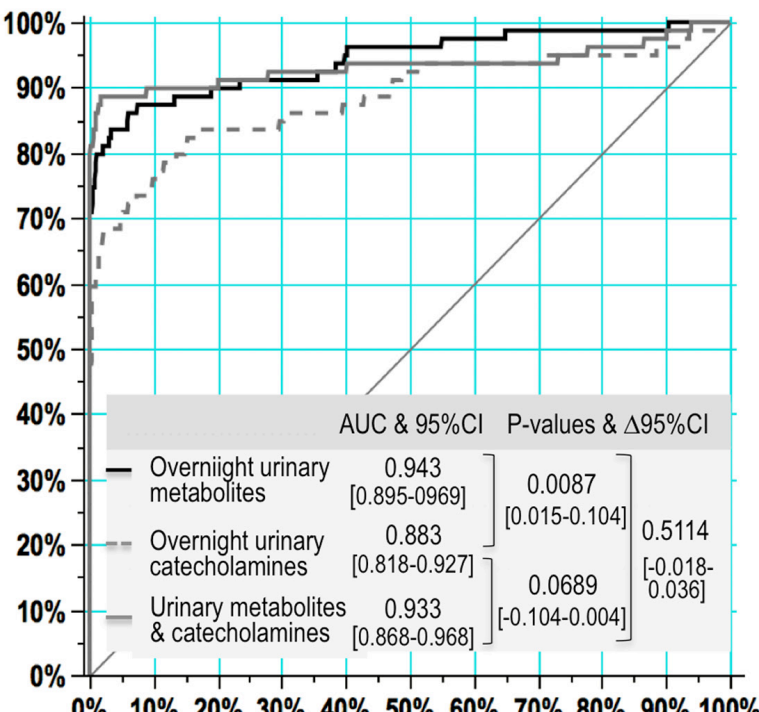

\section{1 - specificity}

Figure 3

Receiver-operating characteristic curves constructed using logistic regression for plasma concentrations vs urinary outputs for 24-h and overnight collections of free normetanephrine, metanephrine and methoxytyramine (A) and for overnight urinary free metabolites vs urinary catecholamines and the combination of overnight urinary catecholmines and metabolites (B). Data are shown with areas under ROC curves (AUC) and $95 \% \mathrm{Cls}$ of areas under curves. Significance of differences in AUC are shown with $95 \% \mathrm{Cl}$ of differences between AUC ( $\triangle A \cup C)$. the supine, position results in increased plasma-free normetanephrine and a high rate of false positives (5, $22,23,24,25)$. Although higher cut-offs of reference intervals established from seated than supine sampling may minimize the false positives $(24,26)$, this is at the expense of compromised diagnostic sensitivity $(5,7)$. It is therefore important to minimize impacts of increased sympathoadrenal activity for both establishing reference intervals and diagnostic testing.

The concept that reduced sympathoadrenal activity during sleep might result in improved diagnostic performance for tests of catecholamine excess involving urine collected overnight compared to a full $24-\mathrm{h}$ is not new. This idea was originally proposed in two reports in 1975 and 1979, each involving six patients with pheochromocytoma (27, 28). These studies were shortly followed by another study involving 16 patients with pheochromocytoma and a comparison group of 166 hypertensives (29). All 16 patients with pheochromocytoma had values for overnight urinary norepinephrine above upper cut-offs compared to 14 out of 16 who showed elevations for 24-h collections. Diagnostic specificity for overnight collections, at $98 \%$, was also improved compared to the $82 \%$ specificity for 24 -h collections. The authors concluded that 'suppression of norepinephrine and epinephrine excretion during sleep in patients without pheochromocytoma provides a collection period that avoids the influence of stress or postural changes on catecholamine excretion'.

The present findings that day-to-night decreases of urinary-free normetanephrine and metanephrine among subjects without PPGL were proportionally smaller than decreases of the parent catecholamines are consistent with previous findings of differences in responsiveness of catecholamines and their O-methylated metabolites to sympathoadrenal activation (30). In particular, the smaller day-to-night decrease in metanephrine than epinephrine is explained by the predominant source of metanephrine from epinephrine continuously leaking from storage vesicles into the cytoplasm of adrenal medullary cells, a source that is independent of epinephrine secretion from those cells (31). Thus, any advantage of overnight compared to 24-h urine collections is minimal for metanephrine, though substantial for epinephrine. In contrast, the larger component of normetanephrine derived from metabolism of norepinephrine released by sympathetic nerves compared to metabolism within adrenal medullary cells does provide a theoretical 
Table 3 Diagnostic test performance of conventional testing strategies of plasma-free metabolites or 24-h urinary outputs of free metabolites compared to daytime and overnight urine collections normalized to creatinine. Data are shown as means with $95 \% \mathrm{Cls}$ in brackets and numbers of positive or negative results relative to the sum of positive and negative results in parentheses.

\begin{tabular}{lc}
\hline & Sensitivity \\
\hline Conventional testing strategies & $97.5(93.9-100.0) \%(77 / 79)$ \\
Plasma-free metabolites & $87.3(79.8-94.8) \%(69 / 79)^{\dagger}$ \\
24-h urinary-free metabolites & $89.9(83.1-96.7) \%(71 / 79)^{\dagger}$ \\
Daytime and overnight strategies according to respective daytime and overnight upper cut-offs \\
Daytime urinary-free metabolites & $93.7(88.2-99.2) \%(74 / 79)^{\S}$ \\
Overnight urinary-free metabolites & $93.7(88.2-99.2) \%(74 / 79)^{\S}$ \\
Daytime and overnight strategies according to optimized universal day/night upper cut-offs \\
Daytime urinary-free metabolites & $93.7(88.2-99.2) \%(74 / 79)^{\S}$ \\
Overnight urinary-free metabolites &
\end{tabular}

\begin{tabular}{l}
\hline Specificity \\
\hline $94.7(93.0-96.5) \%(594 / 627)$ \\
$94.7(93.0-96.5) \%(594 / 627)$ \\
$92.0(89.9-94.1) \%(577 / 627)^{\star \star q}$ \\
$87.6(85.0-90.2) \%(549 / 627)^{\star *}$ \\
$88.2(85.7-90.7) \%(553 / 627)^{\star *}$ \\
$94.3(92.4-96.1) \%(591 / 627)^{\star * *}$ \\
\hline
\end{tabular}

${ }^{a}$ Test performance established using upper cut-offs stipulated in Table 2 for both day and night and sex-specific reference intervals; ${ }^{\mathrm{b} T e s t}$ performance established using sex-specific universal upper cut-offs for combined day and night collections as stipulated in Table 2 ; ${ }^{\dagger} P<0.02$, lower sensitivity than plasma test; ${ }^{\circledR} P<0.05$ higher sensitivity than 24 -h urine test; ${ }^{*} P<0.05$ and $* * P<0.0001$, lower specificity than plasma and 24 -h urine tests; ${ }^{\Pi P} P<0.002$, higher specificity than the corresponding overnight test and other daytime test; $* \star * P<0.0001$, higher specificity than corresponding daytime test. The significance of differences in sensitivity and specificity were established by paired analyses (McNemar's test). See Supplementary Table 1 for differences between tests, $95 \% \mathrm{Cls}$ of differences between tests and exact $P$-values.

advantage for measurements of normetanephrine in overnight compared to 24 -h urine collections.

Thus, our study builds on concepts introduced in earlier studies $(27,28,29)$, but with a focus on urinary-free metanephrines and methoxytyramine, which provide superior accuracy for diagnosis of PPGLs compared to the parent catecholamines and improved specificity compared to the deconjugated metabolites (4). Compared to those earlier studies, our study also includes larger groups of patients in whom PPGLs were excluded and confirmed, as well as an appropriately sized reference population for independently establishing cutoffs. Although ROC curves showed similar diagnostic performance for measurements of free metabolites in overnight and 24-h urine collections, with appropriately chosen reference intervals, diagnostic sensitivity was higher at similar specificities for measurements performed in overnight compared to 24 -h urine collections. This provides some support for suggestions that there may be a diagnostic advantage in assessing catecholamine excess in patients with PPGLs using overnight compared to 24-h urine collections $(27,28,29)$. Also, diagnostic sensitivities were similar but specificities higher for measurements of metabolites in overnight than daytime collections, at least when using the same reference intervals for both; this suggests that there may also be diagnostic advantages in first-morning urine collections compared to collections performed at other times during the day.

Despite supporting the concepts introduced in earlier studies $(27,28,29)$, our study does not establish that measurements of the free metabolites in overnight/ first-morning urine samples offer similar diagnostic accuracy to measurements performed in plasma using collections of blood after supine rest and an overnight fast. In part, this may reflect superiority of measurements of methoxytyramine in plasma compared to urine for detection of dopamine-producing tumors $(4,32)$. This superiority reflects derivation of most dopamine and methoxytyramine in urine from renal decarboxylation of circulating L-dopa $(33,34)$, which potentially contributes to the lack of day-to-night differences in urinary excretion of dopamine and methoxytyramine.

Diagnostic superiority of measurements of metabolites in plasma compared to overnight urine collections may additionally reflect nocturnal sympathoadrenal activation in some patients. This possibility is suggested by findings that patients in whom PPGLs were excluded exhibited more than 2-fold larger proportions of overnight increases rather than decreases in urinary excretion of norepinephrine (13.2\% vs 3.6\%) and normetanephrine $(15.5 \%$ vs $6.6 \%)$ than in the reference population. This may reflect a higher prevalence of pathological conditions associated with sympathoadrenal activation, such as sleep apnea syndrome, in the patient group than in the healthier volunteers. Such influences are known to blunt or reverse day-to-night falls in sympathoadrenal activity and blood pressure (11) and may thereby contribute to the lower diagnostic accuracy of overnight urinary compared to plasma-free metabolites.

Apart from already outlined strengths and limitations of the PMT study (4), a limitation of the presently described sub-study was that reference intervals for day and night 
urine collections were only established for adults. In children, age-specific reference intervals are essential for urinary catecholamines and metabolites, particularly when normalized to creatinine $(35,36,37)$. The present study included three patients tested for PPGL under the age of 18 , only one of whom had a pheochromocytoma indicated by clearly positive test results. Since 24-h collections of urine in children can be unreliable, spot urine collections are particularly important for this group who should be included in any further studies focusing on first-morning urine collections.

Another limitation of the present study was that split day and night urine collections were carried out in only a third of the over 2000 patients enrolled into the PMT protocol. Whether there is any true diagnostic advantage of overnight compared to $24-\mathrm{h}$ collections requires a further large-scale prospective study. Nevertheless, lack of differences in ROC curve-based measures of diagnostic performance for the various urine measurements does provide evidence to support use of nocturnal urine collections as an alternative to 24 -h collections, thereby minimizing inconvenience to patients and avoiding problems associated with incorrect 24 -h collections.

\section{Supplementary materials}

This is linked to the online version of the paper at https://doi.org/10.1530/ EJE-19-1016.

\section{Declaration of interest}

The authors declare that there is no conflict of interest that could be perceived as prejudicing the impartiality of this study.

\section{Funding}

This work was supported by the Deutsche Forschungsgemeinschaft (DFG, German Research Foundation Project number 314061271-CRC/TRR 205/1).

\section{STARD}

Standards for Reporting Diagnostic accuracy are detailed in the supplement.

\section{References}

1 Lenders JWM, Duh QY, Eisenhofer G, Gimenez-Roqueplo AP, Grebe SK, Murad MH, Naruse M, Pacak K, Young WF \& Endocrine Society. Pheochromocytoma and paraganglioma: an Endocrine Society Clinical Practice Guideline. Journal of Clinical Endocrinology and Metabolism 201499 1915-1942. (https://doi.org/10.1210/ jc.2014-1498)

2 Eisenhofer G. Free or total metanephrines for diagnosis of pheochromocytoma: what is the difference? Clinical Chemistry 2001 47 988-989. (https://doi.org/10.1093/clinchem/47.6.988)
3 Pamporaki C, Darr R, Bursztyn M, Glockner S, Bornstein SR, Lenders JW, Pacak K, Krinner A \& Eisenhofer G. Plasma-free vs deconjugated metanephrines for diagnosis of phaeochromocytoma. Clinical Endocrinology 201379 476-483. (https://doi.org/10.1111/ cen.12191)

4 Eisenhofer G, Prejbisz A, Peitzsch M, Pamporaki C, Masjkur J, Rogowski-Lehmann N, Langton K, Tsourdi E, Peczkowska M, Fliedner $\mathrm{S}$ et al. Biochemical diagnosis of chromaffin cell tumors in patients at high and low risk of disease: plasma versus urinary free or deconjugated O-methylated catecholamine metabolites. Clinical Chemistry 201864 1646-1656. (https://doi.org/10.1373/ clinchem.2018.291369)

5 Lenders JW, Willemsen JJ, Eisenhofer G, Ross HA, Pacak K, Timmers HJ \& Sweep CG. Is supine rest necessary before blood sampling for plasma metanephrines? Clinical Chemistry $2007 \mathbf{5 3}$ 352-354. (https://doi.org/10.1373/clinchem.2006.076489)

6 Darr R, Pamporaki C, Peitzsch M, Miehle K, Prejbisz A, Peczkowska M, Weismann D, Beuschlein F, Sinnott R, Bornstein SR et al. Biochemical diagnosis of phaeochromocytoma using plasmafree normetanephrine, metanephrine and methoxytyramine: importance of supine sampling under fasting conditions. Clinical Endocrinology 201480 478-486. (https://doi.org/10.1111/cen.12327)

7 Darr R, Kuhn M, Bode C, Bornstein SR, Pacak K, Lenders JWM $\&$ Eisenhofer G. Accuracy of recommended sampling and assay methods for the determination of plasma-free and urinary fractionated metanephrines in the diagnosis of pheochromocytoma and paraganglioma: a systematic review. Endocrine 201756 495-503. (https://doi.org/10.1007/s12020-017-1300-y)

8 Griffin TP, Bogdanet D, Navin P, Callagy G, O'Shea PM \& Bell M. The importance of standardisation of measurement and reference intervals for detection of phaeochromocytoma and paraganglioma (PPGL). Irish Journal of Medical Science 2018187 993-998. (https:// doi.org/10.1007/s11845-018-1756-7)

9 de Jong WH, Eisenhofer G, Post WJ, Muskiet FA, de Vries EG \& Kema IP. Dietary influences on plasma and urinary metanephrines: implications for diagnosis of catecholamine-producing tumors. Journal of Clinical Endocrinology and Metabolism 200994 2841-2849. (https://doi.org/10.1210/jc.2009-0303)

10 Dodt C, Breckling U, Derad I, Fehm HL \& Born J. Plasma epinephrine and norepinephrine concentrations of healthy humans associated with nighttime sleep and morning arousal. Hypertension 199730 71-76. (https://doi.org/10.1161/01.hyp.30.1.71)

11 Marrone O, Riccobono L, Salvaggio A, Mirabella A, Bonanno A \& Bonsignore MR. Catecholamines and blood pressure in obstructive sleep apnea syndrome. Chest 1993103 722-727. (https://doi. org/10.1378/chest.103.3.722)

12 Sherwood A, Steffen PR, Blumenthal JA, Kuhn C \& Hinderliter AL. Nighttime blood pressure dipping: the role of the sympathetic nervous system. American Journal of Hypertension 200215 111-118. (https://doi.org/10.1016/s0895-7061(01)02251-8)

13 Rasch B, Dodt C, Molle M \& Born J. Sleep-stage-specific regulation of plasma catecholamine concentration. Psychoneuroendocrinology 2007 32 884-891. (https://doi.org/10.1016/j.psyneuen.2007.06.007)

14 Peitzsch M, Prejbisz A, Kroiss M, Beuschlein F, Arlt W, Januszewicz A, Siegert G \& Eisenhofer G. Analysis of plasma 3-methoxytyramine, normetanephrine and metanephrine by ultraperformance liquid chromatography-tandem mass spectrometry: utility for diagnosis of dopamine-producing metastatic phaeochromocytoma. Annals of Clinical Biochemistry 201350 147-155. (https://doi.org/10.1258/ acb.2012.012112)

15 Peitzsch M, Adaway JE \& Eisenhofer G. Interference from 3-O-methyldopa with ultra-high performance LC-MS/MS measurements of plasma metanephrines: chromatographic separation remains important. Clinical Chemistry 201561 993-996. (https://doi.org/10.1373/clinchem.2015.239962) 
16 Peitzsch M, Pelzel D, Glockner S, Prejbisz A, Fassnacht M, Beuschlein F, Januszewicz A, Siegert G \& Eisenhofer G. Simultaneous liquid chromatography tandem mass spectrometric determination of urinary free metanephrines and catecholamines, with comparisons of free and deconjugated metabolites. Clinica Chimica Acta: International Journal of Clinical Chemistry 2013418 50-58. (https:// doi.org/10.1016/j.cca.2012.12.031)

17 Eisenhofer G, Peitzsch M, Kaden D, Langton K, Mangelis A, Pamporaki C, Masjkur J, Geroula A, Kurlbaum M, Deutschbein T et al. Reference intervals for LC-MS/MS measurements of plasma free, urinary free and urinary acid-hydrolyzed deconjugated normetanephrine, metanephrine and methoxytyramine. Clinica Chimica Acta: International Journal of Clinical Chemistry 2019490 46-54. (https://doi.org/10.1016/j.cca.2018.12.019)

18 Chortis V, Bancos I, Crowley RK \& Arlt W. Supine or sitting? Economic considerations regarding patient position during plasma metanephrine analysis for the exclusion of chromaffin tumours. Clinical Endocrinology 201582 462-463. (https://doi.org/10.1111/ cen.12587)

19 Esler MD \& Nestel PJ. Sympathetic responsiveness to head-up tilt in essential hypertension. Clinical Science 197344 213-226. (https://doi. org/10.1042/cs0440213)

20 Young JB, Rowe JW, Pallotta JA, Sparrow D \& Landsberg L. Enhanced plasma norepinephrine response to upright posture and oral glucose administration in elderly human subjects. Metabolism: Clinical and Experimental 198029 532-539. (https://doi.org/10.1016/00260495(80)90078-5)

21 Eisenhofer G, Whiteside EA \& Johnson RH. Plasma catecholamine responses to change of posture in alcoholics during withdrawal and after continued abstinence from alcohol. Clinical Science 198568 71-78. (https://doi.org/10.1042/cs0680071)

22 Kim HJ, Lee JI, Cho YY, Lee SY, Kim JH, Jung BC, Kim SW, Chung JH, Min YK, Lee MS et al. Diagnostic accuracy of plasma free metanephrines in a seated position compared with 24-hour urinary metanephrines in the investigation of pheochromocytoma. Endocrine Journal 201562 243-250. (https://doi.org/10.1507/endocrj.EJ140384)

23 Boot C, Toole B, Johnson SJ, Ball S \& Neely D. Single-centre study of the diagnostic performance of plasma metanephrines with seated sampling for the diagnosis of phaeochromocytoma/paraganglioma. Annals of Clinical Biochemistry 201754 143-148. (https://doi. org/10.1177/0004563216650463)

24 Casey R, Griffin TP, Wall D, Dennedy MC, Bell M \& O'Shea PM. Screening for phaeochromocytoma and paraganglioma: impact of using supine reference intervals for plasma metanephrines with samples collected from fasted/seated patients. Annals of Clinical Biochemistry 201754 170-173. (https://doi. org/10.1177/0004563216646395)

25 Boyd J, Leung AA, Sadrzadeh HS, Pamporaki C, Pacak K, Deutschbein T, Fliedner S \& Kline GA. A high rate of modestly elevated plasma normetanephrine in a population referred for suspected PPGL when measured in a seated position. European Journal of Endocrinology 2019181 301-309. (https://doi.org/10.1530/EJE-190176)
26 Eisenhofer G, Darr R, Pamporaki C, Peitzsch M, Bornstein S \& Lenders JW. Supine or Sitting? Economic and other considerations for use of plasma metanephrines for diagnosis of phaeochromocytoma. Clinical Endocrinology 201582 463-464. (https://doi.org/10.1111/cen.12602)

27 Sullivan JM \& Solomon HS. The diagnosis of pheochromocytoma. Overnight excretion of catecholamine metabolites. JAMA 1975231 618-619. (https://doi.org/10.1001/jama.1975.03240180054017)

28 Ganguly A, Henry DP, Yune HY, Pratt JH, Grim CE, Donohue JP \& Weinberger MH. Diagnosis and localization of pheochromocytoma. Detection by measurement of urinary norepinephrine excretion during sleep, plasma norepinephrine concentration and computerized axial tomography (CT-scan). American Journal of Medicine 197967 21-26. (https://doi.org/10.1016/00029343(79)90064-0)

29 Peaston RT, Lennard TW \& Lai LC. Overnight excretion of urinary catecholamines and metabolites in the detection of pheochromocytoma. Journal of Clinical Endocrinology and Metabolism 199681 1378-1384. (https://doi.org/10.1210/jcem.81.4.8636337)

30 Eisenhofer G, Friberg P, Pacak K, Goldstein DS, Murphy DL, Tsigos C, Quyyumi AA, Brunner HG \& Lenders JW. Plasma metadrenalines: do they provide useful information about sympatho-adrenal function and catecholamine metabolism? Clinical Science 199588 533-542. (https://doi.org/10.1042/cs0880533)

31 Eisenhofer G, Huynh TT, Hiroi M \& Pacak K. Understanding catecholamine metabolism as a guide to the biochemical diagnosis of pheochromocytoma. Reviews in Endocrine and Metabolic Disorders 20012 297-311. (https://doi.org/10.1023/a:1011572617314)

32 Eisenhofer G, Goldstein DS, Sullivan P, Csako G, Brouwers FM, Lai EW, Adams KT \& Pacak K. Biochemical and clinical manifestations of dopamine-producing paragangliomas: utility of plasma methoxytyramine. Journal of Clinical Endocrinology and Metabolism 200590 2068-2075. (https://doi.org/10.1210/jc.20042025)

33 Baines AD, Craan A, Chan W \& Morgunov N. Tubular secretion and metabolism of dopamine, norepinephrine, methoxytyramine and normetanephrine by the rat kidney. Journal of Pharmacology and Experimental Therapeutics 1979208 144-147.

34 Brown MJ \& Allison DJ. Renal conversion of plasma DOPA to urine dopamine. British Journal of Clinical Pharmacology 198112 251-253. (https://doi.org/10.1111/j.1365-2125.1981.tb01210.x)

35 Fitzgibbon MC \& Tormey WP. Paediatric reference ranges for urinary catecholamines/metabolites and their relevance in neuroblastoma diagnosis. Annals of Clinical Biochemistry 199431 1-11. (https://doi. org/10.1177/000456329403100101)

36 Pussard E, Neveux M \& Guigueno N. Reference intervals for urinary catecholamines and metabolites from birth to adulthood. Clinical Biochemistry 200942 536-539. (https://doi.org/10.1016/j. clinbiochem.2008.10.022)

37 Davidson DF, Hammond PJ, Murphy DL \& Carachi R. Agerelated medical decision limits for urinary free (unconjugated) metadrenalines, catecholamines and metabolites in random urine specimens from children. Annals of Clinical Biochemistry 201148 358-366. (https://doi.org/10.1258/acb.2011.011023)
Received 12 December 2019

Revised version received 11 March 2020

Accepted 18 March 2020 